\title{
IDENTIFICAÇÃO DE PROBLEMAS VOCAIS ENFRENTADOS POR CANTORES DE IGREJA
}

\section{Identification of vocal problems experienced by church singers}

\author{
Vanessa Veis Ribeiro ${ }^{(1)}$, Angelika Bissolotti dos Santos ${ }^{(2)}$, Eveline Bonki ${ }^{(3)}$, \\ Tatiane Prestes ${ }^{(4)}$, Ana Paula Dassie-Leite ${ }^{(5)}$
}

\begin{abstract}
RESUMO
Objetivo: identificar possíveis problemas vocais enfrentados por cantores evangélicos de Irati-PR. Método: foram analisados 42 questionários respondidos por cantores de Igreja, onde constavam dados de identificação e um questionário referente a 30 problemas vocais que poderiam ser enfrentados pelos cantores, tanto referentes aos problemas de saúde vocal, quanto relacionados à utilização específica da voz cantada. O questionário já é amplamente utilizado na literatura brasileira. Resultados: a média de problemas referidos foi de 7,78 . Mulheres referiram maior quantidade média de problemas vocais (média:9,65) do que homens (média:6,19), havendo diferença estatisticamente significante entre eles $(p=0,03)$. Das 30 questões, apenas uma não foi assinalada por nenhum cantor. Os problemas vocais mais citados foram: dificuldades para atingir notas agudas ou graves $(n=29 ; 58 \%)$, falta de ar para terminar frases musicais $(n=27 ; 54 \%)$, sensação de aperto ou bola na garganta ( $n=25 ; 50 \%)$, sensação de voz fraca ou forte demais para o canto coral $(n=22 ; 44 \%)$, e desafinação $(n=18 ; 42 \%)$. Além disso, foi possível observar que não houve aumento de problemas vocais com o avanço da idade ou do tempo de canto ( $p=0,003$ e $p=0,573$, respectivamente). Conclusão: mulheres referiram mais problemas do que homens no que se refere à utilização da voz no canto coral. A quantidade de problemas vocais independeu da idade e do tempo de canto, o que pode indicar que a falta de técnica pode ser prejudicial até mesmo para indivíduos que cantam há pouco tempo. As queixas mais frequentes parecem ter maior relação com a falta de técnica vocal.
\end{abstract}

DESCRITORES: Disfonia; Distúrbios da voz; Qualidade da voz; Voz; Música

\section{INTRODUÇÃO}

A voz é o som mais complexo e sofisticado produzido pelo nosso corpo, sendo usada para muitos fins, como a fala e o canto, sendo esta última a forma mais antiga e natural de se produzir

(1) Acadêmica do curso de Fonoaudiologia da Universidade Estadual do Centro-Oeste - UNICENTRO, Irati, PR, Brasil.

(2) Acadêmica do curso de Fonoaudiologia da Universidade Estadual do Centro-Oeste - UNICENTRO, Irati, PR, Brasil.

(3) Acadêmica do curso de Fonoaudiologia da Universidade Estadual do Centro-Oeste - UNICENTRO, Irati, PR, Brasil.

(4) Acadêmica do curso de Fonoaudiologia da Universidade Estadual do Centro-Oeste - UNICENTRO, Irati, PR, Brasil.

(5) Fonoaudióloga; Professora Assistente do Departamento de Fonoaudiologia da Universidade Estadual do Centro Oeste - UNICENTRO, Irati, PR, Brasil; Mestre em Saúde da Criança e do Adolescente pela Universidade Estadual de Campinas.

Conflito de interesses: inexistente música ${ }^{1}$. Quanto às diferenças entre a fala e o canto, a voz falada requer relativamente pouca movimentação pulmonar, ressonância geralmente média, seu ritmo e velocidade geralmente acontecem sem a consciência do falante, sua qualidade é sensível ao interlocutor assim como a outros fatores da situação de comunicação, e seu objetivo normalmente é apenas a comunicação. Já a voz cantada requer relativamente grande movimentação pulmonar, possui ressonância geralmente na "máscara", seu ritmo e velocidade são controlados e ensaiados, sua qualidade depende do treino do cantor, e seu objetivo pode ser, além da comunicação, artístico ou lúdico².

Cantar alivia as tensões e tristezas do dia-a-dia, é uma forma de terapia, de explorar e dar sentido à vida, o que justifica o fato de muitas igrejas possuírem grupos de canto de louvor ${ }^{3}$. Estes grupos, em geral, são formados por cantores e músicos que 
fazem uso da música para conduzir a comunidade durante o culto para o canto e a adoração, e dão à voz uma grande importância em sua relação com Deus, sendo ela um dos meios pelo qual expressam suas emoções, fazem agradecimentos e pedidos ${ }^{4}$.

Grande parte dos corais, tanto de igrejas, escolas e corais municipais, entre outros estabelecimentos, é formada por cantores amadores. Esses, por serem amadores, não obtêm dinheiro ou fama com o canto, fazem geralmente por prazer e lazer ${ }^{5}$. Por isso, é comum que não possuam noções básicas sobre a produção da voz e conseqüentemente não conheçam na totalidade os fatores que contribuem para uma boa saúde vocal ${ }^{6}$. Essa falta de conhecimento pode desencadear em uso excessivo e inadequado da voz, com conseqüentes problemas de atrito vocal, redução das capacidades vocais e desgaste do mecanismo vocal ${ }^{7}$.

Em estudo envolvendo corais evangélicos amadores, observou-se que normalmente não há seleção para a entrada de pessoas no coral, ou seja, mesmo se o indivíduo não tem aptidão ou se ele apresenta alguma alteração vocal, ele é admitido para o grupo. Isso ocorre porque, na maioria das vezes, somente é levado em consideração a vontade de servir e adorar a Deus, não excluindo ninguém dessa vontade própria ${ }^{8}$. Esses aspectos relacionados à seleção, dentre outros fatores, podem ter relação com o fato de que nos últimos tempos vêm aumentando a procura dos cantores de igreja por atendimento especializado. Assim, é importante que o fonoaudiólogo identifique os possíveis problemas vocais enfrentados pelos cantores e possa desenvolver programas e ações em beneficio deste grupo de pessoas ${ }^{9}$.

Já existe a difusão da informação de que o fonoaudiólogo é o profissional responsável por transmitir orientações sobre saúde vocal aos profissionais da voz. Além disso, alguns hábitos já são conhecidos por grande parte das pessoas, como a importância da hidratação ${ }^{10}$. No entanto, acredita-se, aqui, que cada grupo possui suas especificidades e necessidades em relação à saúde vocal e conhecer os problemas enfrentados por cada grupo auxilia no planejamento das ações.

Sendo assim, o objetivo deste trabalho é identificar possíveis problemas vocais enfrentados por cantores evangélicos de Irati-PR.

\section{MÉTODO}

Foi realizado um estudo transversal, de caráter descritivo exploratório e natureza quantitativa com uma população de 42 cantores de diferentes igrejas evangélicas localizadas cidade de Irati-PR, com idade entre 18 e 60 anos (média de 33 anos), de ambos os sexos, sendo 22 homens e 20 mulheres. A atividade de canto em igreja dos cantores variou de 8 meses a 60 anos (média de 11,16 anos). Os cantores responderam a um questionário (Figura 1) que contém 30 afirmativas referentes a possíveis problemas vocais enfrentados por cantores ${ }^{2}$. Tal instrumento foi criado para que os cantores percebam e detectem problemas relacionados à utilização da voz cantada. As afirmações referemse tanto a problemas mais gerais de utilização da voz e saúde vocal (exemplos:"você apresenta quadros alérgicos nas vias respiratórias" e "você tem resfriados, gripes, amidalites, faringites ou laringites freqüentes"), quanto a problemas específicos relacionados à atividade de canto (exemplos: "você desafina ou perde o controle da emissão" e "você sente dificuldades no pianíssimo ou no fortíssimo").

Os cantores foram orientados a assinalar o espaço específico em frente às afirmativas, caso o problema vocal descrito seja enfrentado por eles. Era possível que o participante assinalasse quantas afirmativas/problemas julgasse condizentes com sua situação vocal. Além do questionário, foram extraídos também dados de identificação pessoal, incluindo sexo, idade, profissão, tipo de canto (coro, grupo vocal, solo, etc.) e tempo de utilização da voz cantada.

Foram incluídos na pesquisa cantores amadores com pelo menos um ano de participação em coros, grupos vocais, canto solo ou outras modalidades de canto na igreja que frequentam e que aceitaram participar voluntariamente da pesquisa. Foram excluídos indivíduos que já tivessem passado por processo terapêutico fonoaudiólogico anterior devido a problema vocal.

A pesquisa foi realizada respeitando-se a resolução 196/96 do Conselho Nacional de Saúde / CONEP. Não houve riscos para os sujeitos e todos os participantes assinaram o Termo de Consentimento Livre e Esclarecido (TCLE). A pesquisa foi aprovada pelo Comitê de Ética em Pesquisa da Universidade Estadual do Centro-Oeste, sob o número 252010.

Os dados foram coletados em locais diversos (visitas domiciliares a cantores conhecidos, ao final de cultos, ensaios, ao final de apresentações em locais públicos, etc.), sendo os mesmos tabulados e posteriormente realizou-se a seleção das variáveis de acordo com os objetivos da pesquisa. Os dados foram analisados estatisticamente por meio dos testes não paramétricos "Igualdade de duas proporções", "ANOVA" e "Teste de Correlação de Pearson". Foi adotado um nível de significância de $5 \%(p=0,05)$. 


\section{RESULTADOS}

A Tabela 1 mostra a análise descritiva completa dos dados referentes à idade, tempo de atividade de canto e quantidade média de problemas enfrentados pelos cantores de igreja durante a utilização da voz cantada. Observa-se uma média de 7,78 problemas, de uma possibilidade total de 30 .

Na Tabela 2, é possível observar os dados referentes aos problemas vocais mais referidos pelos cantores. Os problemas, relacionado à voz cantada, mais referidos pelos participantes foram "dificuldades para atingir as notas agudas ou graves" $(n=29 ; 58 \%)$, "falta de ar para terminar as frases musicais" ( $n=27 ; 54 \%)$, "possuir alguns sintomas na laringe, como: coceira, ardor, dor, sensação de garganta seca, sensação de queimação, sensação de aperto ou bola na garganta" ( $n=25 ; 50 \%)$, "sentir que a voz é fraca ou forte demais para o canto coral" ( $n=22 ; 44 \%)$, "desafinar ou perder o controle da emissão" ( $n=8 ; 42 \%)$.

A Tabela 3 mostra a relação entre sexo e idade, tempo de atividade de canto e quantidade de problemas referida pelos cantores. Observa-se que as mulheres referem maior quantidade média de problemas (média: 9,65) do que os homens (média: $6,19)$, havendo diferença estatisticamente significante entre eles $(p=0,03)$. Para os outros cruzamentos com a variável sexo, não houve diferença estatisticamente significante.

A Tabela 4 apresenta correlação entre idade e quantidade de problemas vocais, e o tempo de atividade de canto referido pelos cantores. É possível observar que não houve aumento de problemas à medida que houve aumento do tempo de atividade de canto ( $p=0,003$ e $p=0,573$, respectivamente).

\section{Dados de identificação}

Nome:

Sexo:

Tipo de canto: ( ) coral

Canta há quanto tempo:
Idade:

Profissão:

( ) outro:

\section{Assinale a resposta quando esta é POSITIVA:}

( ) Você acha que sua voz é rouca ou alguém já comentou que sua voz é rouca.

( ) Você fica rouco por mais de dois dias sem motivo aparente.

( ) Sua voz fica rouca após ensaios ou apresentações.

( ) Você tem ou já teve algum problema de voz diagnosticado.

( ) Sua voz piorou depois que você começou a cantar.

( ) Ultimamente você tem demorado mais tempo para aquecer a voz.

( ) Durante o canto sua voz quebra ou some.

( ) Você desafina ou perde o controle da emissão.

( ) Você sente dificuldades no pianíssimo ou no fortíssimo.

( ) Você sente que sua voz é fraca ou forte demais para o canto coral

( ) Você tem dificuldades para atingir as notas agudas ou graves.

( ) Você sente alguma instabilidade vocal ao cantar.

( ) Quando você canta percebe que sai "ar" na voz.

( ) Falta ar para você terminar as frases musicais.

( ) Quando você canta suas veias ou músculos do pescoço saltam.

( ) Você canta em diversos naipes ou mudou de naipe recentemente.

( ) Você procura cantar mais forte que os demais componentes do coral.

( ) Você sente dor de cabeça ou na região do pescoço quando canta.

( ) Você dubla certos trechos da música que não consegue cantar.

( ) Você tem alguns desses sintomas na laringe: coceira, ardor, dor, sensação de garganta seca, sensação de queimação, sensação de aperto ou bola na garganta.

( ) Você pigarreia constantemente.

( ) Você apresenta quadros alérgicos nas vias respiratórias.

( ) Você tem resfriados, gripes, amidalites, faringites ou laringites freqüentes.

( ) Você tem dificuldades digestivas, azia ou refluxo gastresofágico.

( ) Você consegue controlar sua emissão cantada no coral.

( ) Seu coral costuma interpretar diversos estilos musicais.

( ) Além do coral, você canta habitualmente em outras situações ou utiliza a voz falada em demasia.

( ) Você canta durante muitas horas seguidas.

( ) Você fuma ou ingere bebidas alcoólicas frequentemente.

( ) Você se automedica quando tem problemas de voz. 
Tabela 1 - Descrição de idade, tempo de canto e quantidade de problemas referidos pelos cantores

\begin{tabular}{lccc}
\hline Descritiva & Idade & Tempo de Canto & Qtde Problemas \\
\hline Média & 33 & 11,16 & 7,78 \\
Mediana & 31 & 8 & 7 \\
Desvio Padrão & 13,93 & 12 & 4,19 \\
\hline
\end{tabular}

Tabela 2 - Distribuição dos cinco problemas de maior ocorrência

\begin{tabular}{lccc}
\hline Problemas referidos pelos cantores & $\mathbf{N}$ & $\%$ & p-valor \\
\hline Dificuldades para atingir notas agudas ou graves & 29 & 58 & 0,737 \\
Falta ar para terminar as frases musicais & 27 & 54 & 0,687 \\
Sintoma na laringe (coceira, ardor, dor, bolo na garganta,...) & 25 & 50 & 0,422 \\
Voz é fraca ou forte demais para o canto coral & 22 & 44 & 0,161 \\
Desafina ou perde o controle da emissão & 21 & 42 & 0,110 \\
\hline
\end{tabular}

Teste Igualdade de Duas Proporções

Tabela 3 - Comparação entre sexo e idade, tempo de canto e quantidade de problemas referidos pelos cantores

\begin{tabular}{lcccccc}
\hline \multirow{2}{*}{ Sexo } & \multicolumn{2}{c}{ Idade } & \multicolumn{2}{c}{ Tempo de Canto (anos) } & \multicolumn{2}{c}{ Qtde problemas } \\
\cline { 2 - 8 } & Fem & Masc & Fem & Masc & Fem & Masc \\
\hline Média & 30,05 & 35,68 & 9,94 & 12,25 & 9,65 & 6,19 \\
Mediana & 27,5 & 35 & 4 & 10 & 10 & 6 \\
Desvio Padrão & 13,61 & 13,98 & 14,4 & 9,64 & 4,51 & 3,19 \\
p-valor & \multicolumn{2}{c}{0,194} & \multicolumn{2}{c}{0,573} & \multicolumn{2}{c}{0,003} \\
\hline
\end{tabular}

Teste Anova

Tabela 4 - Correlação entre idade e quantidade de problemas com o tempo de canto

\begin{tabular}{lccc}
\hline & & Idade & Qtde problemas \\
\cline { 2 - 4 } Tempo de Canto (anos) & corr & $68,70 \%$ & $-9,40 \%$ \\
& p-valor & $<0,001^{*}$ & 0,584 \\
\hline
\end{tabular}

Teste Correlação de Pearson

\section{DISCUSSÃO}

As pessoas que utilizam a voz como instrumento de trabalho atuam em diversas áreas, porém, cada profissão com suas particularidades. Muitos já sabem sobre a importância de se cuidar da saúde vocal, porém, sendo o fonoaudiólogo responsável por difundir as orientações sobre a saúde vocal, é importante conhecer os problemas enfrentados por cada grupo, o que irá auxiliar no planejamento das ações preventivas voltadas para essa população. Sendo assim buscamos com esse estudo investigar os possíveis problemas vocais apresentados por cantores de Igrejas amadores da cidade de Irati-PR relacionados à atividade de canto, contribuir para o conhecimento do perfil dessa população e incentivar ações fonoaudiológicas que sejam referentes as reais necessidades desse grupo.

A quantidade média de problemas relatados pelos participantes é consideravelmente alta, visto que a média foi de 7,78 por participante, de um total de 30 opções de problemas presentes no questionário. De acordo com a literatura, se a média de problemas apresentados pelos indivíduos for maior que três problemas, essa população já pode ser considerada de risco para o desenvolvimento 
de alterações vocais ${ }^{2}$. A grande quantidade de problemas relatados por cada participante pode ser conseqüência da falta de informações dos cantores de Igreja, visto que a maioria deles é voluntário, e exerce a atividade de canto apenas por lazer, não tendo conhecimento sobre as técnicas de canto e os cuidados necessários para cuidar as saúde vocal quando se utiliza da voz cantada.

Quando se busca outros estudos que observaram a média de problemas vocais enfrentados por grupos específicos, de profissionais e não profissionais da voz, encontram-se dados bastante distintos. Um estudo com 190 frequentadores de um Shopping revelou que a maioria dos entrevistados referiu entre 3 e 12 problemas, dentre 21 listados $^{11}$. Em relação ao tipo de sintoma, estudo realizado com 3265 professores de todo o Brasil, $41 \%$ relataram apresentar rouquidão, sendo a média encontrada de 4 problemas $^{12}$. Em pesquisa com operadores de telesserviços de centrais próprias de instituições bancarias, contatou-se que os mesmos apresentam de 1 a 2 problemas vocais ${ }^{13}$. No que se refere especificamente à voz cantada, um estudo que analisou o perfil vocal de coralistas amadores, obteve o dado de que $49 \%$ relata entre 1 e 2 problemas relacionados a voz cantada ${ }^{14}$ e pesquisa envolvendo regentes de corais obteve a média de 3 problemas referida pelos indivíduos do grupo ${ }^{15}$.

Acredita-se que a média elevada de problemas vocais referidos no presente estudo pode ter relação com a falta de orientações sobre bem-estar vocal ou por falta de técnica vocal específica para o canto. Além disso, todos os problemas listados tinham relação direta com a utilização da voz cantada, o que também pode ter contribuído para a média elevada de problemas referidos pelo grupo. $\mathrm{Na}$ literatura fonoaudiológica, alguns estudos com cantores religiosos evidenciam precárias condições de percepção, conhecimento e importância atribuídas à saúde vocal ${ }^{16}$.

A única questão não assinalada por nenhum dos 50 cantores foi a relacionada ao fumo e ingestão de bebidas alcoólicas frequentemente. Isso pode ter ocorrido pelo fato dos sujeitos possuírem restrições religiosas quanto ao etilismo e ao tabagismo. Todos os outras possibilidades de problemas foram citados por pelo menos um participante.

Alguns estudos evidenciam que a maioria dos cantores de Igreja não possuem um preparo vocal adequado e nem acompanhamento fonoaudiológico, assim a maioria destes apresentam queixas em relação a voz cantada, como, dificuldade para atingir determinados tons, cansaço vocal, pigarro, rouquidão, ardor ao cantar, dentre outros ${ }^{2,6,8}$. Muitos desses cantores de igreja pelo fato de serem amadores, não percebem as alterações, ou, dão pouco valor ao perceberem algumas alterações em sua voz. A falta de conhecimento sobre a saúde vocal pode trazer grandes danos a essa população, visto que a maioria deles utiliza-se da voz cantada frequentemente em ensaios do coral, e em apresentações do mesmo. A grande quantidade de alterações relatadas nos mostra a necessidade de ações voltadas para esse grupo de profissionais, que além de proporcionar um diagnóstico precoce das alterações vocais, também previnem distúrbios fonoaudiológicos por meio de orientação profissional ${ }^{17}$. Observamos que quatro dos cinco problemas mais relatados pelos indivíduos são referentes a falta de técnica vocal e apenas um refere-se a problemas laríngeos que poderiam indicar alguma alteração fonoaudiológica.

No presente estudo, mulheres apresentam maior número médio de problemas vocais do que homens. A literatura refere que o sexo feminino é mais predisponente a distúrbios vocais, dado que foi confirmado com sua pesquisa sobre as condições vocais de vendedores, apenas mulheres referiram alterações vocais já diagnosticadas, no passado ou presente ${ }^{13,18}$. É possível que esses resultados tenham relação com as características anatômicas e de menor proporção glótica das laringes femininas ${ }^{19}$.

A tabela 4 evidenciou que não há relação entre a quantidade de problemas e o tempo de atividade de canto. Pesquisa com regentes de coral associam os problemas vocais apresentados pelos participantes ao cuidado com a saúde vocal, sendo assim, o número de alterações vocais apresentadas pelos indivíduos estaria associado ao que elas chamam de "componente básico para uma boa voz cantada", sendo ele composto por dom e técnica ${ }^{15}$.

O canto exige uma grande demanda vocal, sendo necessários ajustes neuromusculares pelo trato vocal para se conseguir uma grande diversidade de efeitos sonoros e por isso, praticar o canto mesmo que amadoramente necessita de cuidados vocais ${ }^{14}$. Além disso, cantores de coral profissional, semi-profissional e amador não estão bem informados quanto aos conhecimentos básicos de cuidados vocais, o que os leva ao uso excessivo, mau uso ou abuso vocal, podendo resultar em alterações vocais ${ }^{14}$.

Tanto as principais queixas apresentadas por cantores, quanto os hábitos nocivos, poderiam ser minimizados significantemente com a orientação do fonoaudiólogo quanto à higiene vocal ${ }^{2}$. Faz-se necessário ressaltar que perante qualquer alteração da voz o profissional deve procurar atendimento médico com otorrinolaringologista e/ou fonoaudió$\operatorname{logo}^{20}$. Pesquisas também chamam a atenção para a importância da realização de ações preventivas 
relacionadas a voz, auxiliando os profissionais da voz a cuidarem da voz que é o seu instrumento precioso de trabalho ${ }^{20,21}$.

\section{CONCLUSÃO}

A partir dos resultados obtidos pode-se concluir que cantores de igreja apresentam uma média elevada de problemas vocais. Mulheres apresentam maior número médio de problemas vocais do que homens. A quantidade de problemas vocais referida não está relacionada ao tempo de atividade de canto que os indivíduos possuem, mas sim, à provável falta de orientações sobre a saúde e técnicas vocais. As queixas estão mais relacionadas à falta de técnica vocal e menos a problemas laríngeos que poderiam indicar alguma alteração fonoaudiológica.

\begin{abstract}
Purpose: to identify possible problems faced by gospel singers of Irati-PR. Method: we analyzed 42 questionnaires answered by singers from the church, which contained identifying information and a questionnaire related to 30 vocal problems that could be faced by the singers, both related to vocal health problems and the specific use of singing voice. The questionnaire is widely used in the Brazilian literature. Results: the average value of these problems was 7.78. Women reported higher average number of vocal problems (mean: 9.65) than men (mean: 6.19). There is a statistically significant difference between them $(p=0.03)$. From the 30 questions, only one was not checked by any singer. The most cited vocal problems were: difficulties to reach high or bass notes $(n=29 ; 58 \%)$, shortness of breath to complete musical phrases $(n=27 ; 54 \%)$, tightness or ball in the throat $(n=$ $25 ; 50 \%)$, feeling weak or too strong voice for choral singing $(n=22 ; 44 \%)$ and discord $(n=18 ; 42 \%)$. Moreover, it was observed that there is no increase of vocal problems as there is increase of age or singing time $(p=0.003$ and $p=0.573$, respectively). Conclusion: women reported more problems than men regarding voice use. The amount of vocal problems does not depend on age and singing time, this may indicate that the lack of technique can be harmful even for subjects who sing recently. The most frequent complaints seem to have more relation to lack of vocal technique.
\end{abstract}

KEYWORDS: Dysphonia; Voice Disorders; Voice Quality; Voice; Music

\section{REFERÊNCIAS}

1. Camargo TF, Barbosa PFA, Teles LCS. Aspectos de religiosidade na saúde vocal de cantores de grupo de louvor. Rev Soc Bras Fonoaudiologia. 2007; 10(3), 359-68.

2. Behlau M, Rehder MI. Higiene Vocal para o Canto Coral. Rio de Janeiro: Revinter, 2008.

3. Bastos PRJ, Ferreira KL, Camargo ZAD, Pinho SMR. Extensão vocal de cantores de coros evangélicos amadores. In: Pinho SMR. Temas em Voz Profissional. Rio de Janeiro: Revinter, 2007.

4. Penteado RZ, Silva CB e Pereira PFA. Aspectos de religiosidade na saúde vocal de cantores de grupo de louvor. Rev CEFAC. 2008; 10(3), 359-68. 5. Mota ACG, Goldenberg M. Aquecimento e Desaquecimento Vocal [monografia]. São Paulo (SP): Centro de Especialização em Fonoaudiologia Clínica; 1998.

6. Behlau MS, Rehder MIBC. Perfil Vocal de Regentes de Coral do Estado de São Paulo. Rev CEFAC. 2008; 10(1): 206-17.
7. Silva MS, Camargo EAA. Perfil vocal dos coralistas amadores do estilo gospel. Anais do $5^{\circ}$ Simpósio de Ensino de Graduação da Universidade Metodista de Piracicaba. Piracicaba: UNIMEP, 2007. Disponível em: http://www.unimep.br/phpg/ mostraacademica/anais/5mostra/backup/4/339.pdf . Acesso em: 03/03/10.

8. Costa, PJBM; Ferreira KL, Camargo ZA; Pinho SMR. Extensão Vocal de Cantores de Coros Evangélicos Amador. Rev CEFAC. 2006; 8(1): 96-106.

9. Leite GCA, Assumpção R, Campiotto AR, Silva MAA. O canto nas igrejas: o estudo do uso vocal dos coralistas e não coralistas. Dist Comun. 2004; 16(2):229-39.

10. Ueda, KH; Santos, LZ; Oliveira, IB. Vinte e cinco anos de cuidados com a voz profissional: avaliando ações. Rev. CEFAC. 2008, 10(4): 557-65.

11. Ferreira LP, Santos JG, Lima MFB. Sintoma vocal e sua provável causa: levantamento de dados em uma população. Rev. CEFAC. 2009; 11(1):110-8. 
12. Behlau, M; Zambon, F; Guerrieri, AC; Roy, N. Panorama epidemiológico sobre a voz do professor no Brasil. In: $17^{\circ}$ Congresso Brasileiro de Fonoaudiologia e $1^{\circ}$ encontro Ibero- Americano de fonoaudiologia, 2009, Salvador-Bahia. Anais... Salvador- Bahia, 2009, p 1511.

13. Dassie-Leite AP, Lourenço L, Behlau M. Correlação entre dados ocupacionais, sintomas e avaliação vocal de operadores de telesserviços. Revista da Sociedade Brasileira de Fonoaudiologia [ no prelo]. 2010

14. Ribeiro LR, Hanayama EM. Perfil Vocal de Coralistas Amadores. Rev. CEFAC. 2005; 7(2): 252-66.

15. Rehder MIBC, Behlau M. Análise vocal perceptivo-auditiva e acústica, falada e cantada de regentes de coral. Pró-Fono. 2008; 20(3):195-200.

16. Lima MFB, Ferreira LP. Sintomas Vocais, Alterações da Qualidade Vocal e Laríngea em Professores: análise de instrumentos [dissertação]. São Paulo (SP): Pontífice Universidade Católica de São Paulo; 2008.
17. Köhle J, Nemr K, Leite GCA, Santos AO, Lehn CN, Chedid HM. Ação de proteção de saúde vocal: perfil da população e correlação entre auto-avaliação vocal, queixas e avaliação fonoaudiológica perceptivo-auditiva e acústica. Rev Distúrbios da Comunicação. 2004; 16(3): 333-41.

18. Ferreira LP, Luciano P, Akutsu CM. Condições de Produção Vocal de Vendedores. Rev. CEFAC. 2008; 10(4): 528-35.

19. Pontes P, Behlau M, Kyrillos L. Glottic configuration and glottic proportion: na attempt to understand the posterior glottic chink. Rev Otol Rhinol Laryngol (Bordeaux). 1994; 115: 261-6.

20. Fortes FSG, Imamura R, Tsuji DH, Sennes LU. Perfil dos profissionais da voz com queixas vocais atendidos em um centro terciário de saúde. Rev Bras Otorrinolaringol. 2007; 73(1):27-31.

21. Fernades AJ, Kayama AG, Östergren EA. O regente moderno e a construção da sonoridade coral. Per Musi. 2006; 13:33-51.

http://dx.doi.org/10.1590/S1516-18462011005000055

RECEBIDO EM: 22/09/2010

ACEITO EM: 23/01/2011

Endereço para correspondência:

Vanessa Veis Ribeiro

Rua Souza Naves, 227, Bairro Jardim Califórnia

Irati - PR

CEP: 84500-000

E-mail: vanessaribeirooo@ hotmail.com 